\title{
Uma Proposta de Modelo para Avaliação da Qualidade da Tradução de Requisitos para Casos de Uso ${ }^{i}$
}

\author{
Fabiana Zaffalon Ferreira Rocha ${ }^{1}$, Elisa Cerri e Cerri ${ }^{1}$, Ricardo Melo Bastos ${ }^{1}$, \\ Marcelo Hideki Yamaguti ${ }^{1}$ \\ ${ }^{1}$ Faculdade de Informática - Pontifícia Universidade Católica do Rio Grande do Sul \\ Av. Ipiranga, 6681 - Prédio 30, Bloco C, CEP: 90619-900 - Porto Alegre - RS \\ \{frocha, ecerri, bastos, yamaguti\}@inf.pucrs.br
}

\begin{abstract}
Resumo. O documento de especificação de requisitos de software (Software Requirements Specification - SRS) é decisivo para o desenvolvimento de um bom produto final. As métricas desempenham um papel essencial na detecção de defeitos dos requisitos, fornecendo meios para a visualização de discrepâncias e identificação de pontos fora de uma situação projetada. Assim, o objetivo deste trabalho é propor um modelo com a finalidade de avaliar a qualidade da tradução de requisitos para casos de uso.
\end{abstract}

\section{Introdução}

Determinar e gerenciar os requisitos são atividades essenciais no processo de desenvolvimento de um software, pois uma vez que os requisitos estejam incorretos, inconsistentes ou incompletos, o sistema provavelmente não atenderá às reais necessidades do cliente [SOMMERVILLE 2005]. Segundo [JACOBSON 1999], um caso de uso é um "documento narrativo que descreve a seqüência de eventos de um ator que usa um sistema para completar um processo". Assim, os casos de uso são artefatos de fácil compreensão para expressar os requisitos funcionais de um sistema.

Os requisitos podem ter diferentes aspectos de qualidade, tais como, consistência, não ambigüidade, etc. As métricas são definidas para medir a qualidade total de um requisito. São aplicadas nos requisitos expressos em linguagem natural e em casos de uso através dos atributos de qualidade [MORA 2003] e desempenham um papel essencial na detecção de defeitos dos requisitos, fornecendo meios para a visualização de discrepâncias e identificação de pontos fora de uma situação projetada [COSTELLO 1995]. Partindo de modelos de qualidade, algumas métricas são derivadas a fim de executar uma avaliação quantitativa de uma especificação de requisitos [FABBRINI 2001].

Este trabalho visa identificar os elementos envolvidos em um modelo de qualidade de requisitos, tais como a estrutura do documento de especificação dos requisitos e os atributos de qualidade. A partir disto, é proposto um modelo para avaliar a qualidade da tradução dos requisitos para casos de uso, bem como a correlação existente entre os atributos dos documentos. Este artigo está organizado da seguinte forma: a seção 1 corresponde a esta introdução. A seção 2 apresenta o referencial teórico para este trabalho. A seção 3 apresenta o modelo de integração apresentando seus elementos e relacionamentos. A seção 4 descreve o modelo proposto desenvolvido e apresenta a obtenção das métricas através da abordagem GQM. A seção 5 apresenta uma breve descrição dos trabalhos relacionados, bem como cada um contribuiu para o desenvolvimento deste trabalho. E, por fim, a seção 6 apresenta a conclusão deste 
trabalho.

\section{Referencial Teórico}

\subsection{Especificação de Requisitos}

Um dos artefatos gerado durante o desenvolvimento de software é o documento de especificação de requisitos software (Software Requirements Specification - SRS). Esse documento descreve o comportamento externo observado e as características esperadas de um sistema de software. [IEEE 1998] propõe um modelo de documento de especificação de requisitos genérico e pretende ser aplicado em uma variada gama de documentos de requisitos. Em geral, nem todas as partes do documento são necessárias para todos os documentos de requisitos. Cada organização deverá adaptar o padrão de acordo com o tipo de sistema que desenvolve.

\subsection{Especificação de Casos de Uso}

Segundo [COCKBURN 2001] a especificação de um Caso de Uso deve considerar: como e quando o Caso de Uso inicia ou termina; as interações com os atores envolvidos; as seqüências normais de execução; as seqüências alternativas ou de exceção. $\mathrm{O}$ autor propõe um template de especificação de caso de uso, adotado neste trabalho, que define os objetivos dos casos de uso bem como os níveis associados com estes objetivos.

\subsection{Qualidade dos Documentos de Requisitos}

Em geral, a qualidade do SRS contribui para o sucesso do software garantindo a relação custo-benefício de um software que atenda às necessidades do usuário. Se o SRS resultante se mostra incompleto ou inconsistente, existem grandes possibilidades do software sofrer as conseqüências deste fato [PRESSMAN 2001].

O padrão IEEE Std 830 -1998 [IEEE 1998] define um conjunto de critérios de qualidade para documentos de especificação de requisitos de software: correção, não ambigüidade, completude, consistência, ordenação por prioridade, verificabilidade, modificabilidade e rastreabilidade. [DAVIS 1993] acrescenta outras características que devem estar presentes no SRS para que o mesmo seja considerado de qualidade.

\subsection{Métricas de qualidade de requisitos}

As métricas são definidas para medir a qualidade total de um requisito e podem ser aplicadas nos requisitos expressos em linguagem natural e em casos de uso [MORA 2003]. Os requisitos podem ter diferentes aspectos de qualidade, tais como, consistência, integralidade, etc. O quadro 1 mostra algumas métricas.

\section{Quadro 1 Métricas de Qualidade do SRS [DAVIS 1993]}




\begin{tabular}{l|l}
\hline \multicolumn{1}{c|}{ Atributos } & \multicolumn{1}{c}{ Fórmula e Interpretação } \\
\hline \multirow{5}{*}{ Completude } & $Q_{1}=n_{u i} /\left(n_{i} X n_{s}\right)$ \\
& $n_{u i}=$ número total de função $n_{i}=$ número total de entrada \\
& $n_{s}=$ número total de estado \\
& Quanto mais próximo de 1, mais completo está. \\
\hline \multirow{5}{*}{ Consistência } & $Q_{3}=n_{c} / n_{r}$ \\
& $n_{c}=$ número de requisitos que são consistentes. \\
& $n_{r}=$ número total de requisitos. \\
& Quanto mais próximo de 1, mais consistente está. \\
\hline \multirow{5}{*}{ Não ambigüidade } & $Q_{4}=n_{u i} / n_{r}$ \\
& $n_{u i}=$ número de requisitos que apresentam única interpretação \\
& $n_{r}=$ número total de requisitos \\
& Quanto mais próximo de 1, menos ambíguo está. \\
\hline
\end{tabular}

Para que a medição seja efetiva, torna-se necessário focá-la nos objetivos a serem alcançados com tais medições. $O$ uso de modelos pode guiar a definição de aplicação de medições a serem realizadas. Um método comumente utilizado é o GQM (Goal-Question-Metrics). A abordagem do método GQM é baseada na premissa de que uma organização para medir deve primeiro especificar os seus próprios objetivos e os objetivos de seus projetos, então deve-se traçar os objetivos para os dados que os definem operacionalmente, e finalmente prover um framework para interpretação dos dados, respeitando os objetivos estabelecidos anteriormente [SOLINGEN 1999].

\section{Modelo de Integração}

Para a identificação dos elementos foi elaborado um modelo conceitual para os elementos que compõem o SRS e outro para os elementos do modelo de caso de uso. A partir da análise destes modelos foi criado um modelo de Integração que apresenta o relacionamento entre os elementos presentes nos modelos conceituais, ou seja, este relacionamento indica quais classes do SRS podem fornecer informações importantes para especificar os casos de uso.

A importância do modelo de integração para este trabalho é permitir a visualização dos relacionamentos entre as classes dos modelos conceituais permitindo verificar quais os cuidados que devem ser tomados no SRS para que seja possível a especificação de casos de uso de qualidade.

Devido à limitação de espaço não é possível ilustrar o modelo de integração, porém o quadro 2 mostra o relacionamento entre as classes do modelo conceitual gerado a partir do SRS e da especificação de casos de uso.

Quadro 2 Relacionamento das classes no modelo de integração

\begin{tabular}{l|l}
\hline Classes da Especificação de Casos de Uso & Classes do SRS \\
\hline Nome & IntroduçãoRF \\
\hline EscopoUC & EscopoSRS \\
\hline Contexto & FuncoesProduto \\
\hline Participante & IntroducaoRF \\
\hline Stakeholders & FuncoesProduto \\
\hline PreCondições & Processamento \\
\hline GarantiasMinimas & Função Produto e IntroducaoRF \\
\hline GarantiasSucesso & IntroducaoRF e Saida \\
\hline CenarioSucessoPrincipal & Entrada e FuncoesProduto \\
\hline FluxoAlternativo & RespostaSituacoesAnormais \\
\hline Variações & Processamento e FuncoesProduto \\
\hline InformacoesRelacionadas & Atributo e Performance \\
\hline
\end{tabular}

Através de uma aplicação constatamos que a presença de alguns elementos do SRS identificados no quadro acima não são obrigatórias tais como: Entrada, Saída,

Anais do III Simpósio Brasileiro de Sistemas de Informação. Curitiba, PR, novembro de 2006. 
Processamento, Resposta a Situações Anormais, Atributo e Performance, entretanto estas informações contribuem no entendimento de cada requisito bem como na tradução dos mesmos para casos de uso.

Os modelos conceituais do SRS, Modelo de Caso de Uso e Integração foram trabalhos realizados dentro do grupo de Engenharia de Requisitos do Centro de Desenvolvimento e Pesquisas DELL/PUCRS (CDPe).

\section{Modelo de Avaliação da Qualidade da Transcrição de Requisitos para Casos de Uso}

Um dos objetivos do modelo proposto é identificar quais os elementos do SRS são necessários para especificar casos de uso consistentes visando à qualidade na transcrição. Estas informações foram identificadas através do trabalho desenvolvido por [BELGAMO 2005] e através da análise do modelo de integração, considerando os relacionamentos dos elementos dos modelos conceituais do SRS e especificação de casos de uso.

De acordo com [BELGAMO 2005], os campos Introdução, Funções do Produto, Requisitos Funcionais e Atributos são suficientes para extrair as informações necessárias para gerar os casos de uso, entretanto conforme o estudo realizado no modelo de integração e através de uma aplicação verificou-se que outras informações (seção 3) também são importantes para facilitar a transcrição.

\subsection{Atributos de Qualidade do Modelo}

Os atributos adotados para o modelo proposto são os mesmos apresentados na literatura, porém com abordagens diferentes. Enquanto que na literatura o foco está na qualidade do documento em si, avaliando cada requisito individualmente, o foco deste trabalho está na qualidade da transcrição dos requisitos para especificação de casos de uso, ou seja, na qualidade da tradução do requisito para caso de uso correspondente.

No quadro 3 está a relação dos atributos identificados na literatura que são utilizados no trabalho, bem como suas definições e adaptações para a avaliação da qualidade de tradução do modelo proposto.

\section{Quadro 3 Comparação entre os atributos}

\begin{tabular}{l|l|l}
\hline \multicolumn{1}{c|}{ Atributo } & \multicolumn{1}{c|}{ Literatura [DAVIS 1993] } & \multicolumn{1}{c}{ Adaptação } \\
\hline & $\begin{array}{l}\text { Um SRS é completo se descreve todas as demandas } \\
\text { de interesse dos usuários; mostra todas as respostas } \\
\text { esperadas do sistema; se todas as páginas estiverem } \\
\text { numeradas; todas as figuras e tabelas estiverem } \\
\text { nomeadas e numeradas; todos os termos definidos e } \\
\text { todo material presente referenciado. }\end{array}$ & $\begin{array}{l}\text { A transcriça será completa se todos os } \\
\text { elementos presentes no requisito como } \\
\text { por exemplo, atores e funcionalidades } \\
\text { estiverem também presentes no caso de } \\
\text { uso correspondente. }\end{array}$ \\
\hline $\begin{array}{l}\text { Não } \\
\text { ambigüidade }\end{array}$ & $\begin{array}{l}\text { Um SRS é não ambíguo de todos os requisitos } \\
\text { presentes no documento tiverem uma única } \\
\text { interpretação. }\end{array}$ & $\begin{array}{l}\text { Os casos de uso e os requisitos } \\
\text { correspondentes devem ter a mesma } \\
\text { interpretação. }\end{array}$ \\
\hline Consistência & $\begin{array}{l}\text { Um SRS é consistente se não existir contradições } \\
\text { entre os requisitos presentes no documento. }\end{array}$ & $\begin{array}{l}\text { Capacidade de não existir contradição } \\
\text { entre os casos de uso e os requisitos } \\
\text { correspondentes. }\end{array}$ \\
\hline
\end{tabular}

\subsection{Métricas}

Para guiar a seleção dos elementos que irão compor o modelo de medição, o trabalho seguiu a seqüência de passos proposta pelo método GQM. 
$\mathrm{Na}$ fase de planejamento, foram definidos os atributos de qualidade que serão medidos (seção 4.1). Como este trabalho está focado na tradução dos documentos do SRS para especificação dos casos de uso, o principal objetivo que se pretende atingir é a avaliação da qualidade da transcrição dos documentos, onde as métricas que medem a qualidade do documento de especificação de requisitos foram adaptadas para avaliar a qualidade da tradução dos requisitos para casos de uso.

\subsubsection{Definição}

Seguindo o GQM, foram definidos os objetivos de medição. A partir desses objetivos, a seqüência de passos proposta pelo método GQM foi executada. Para cada meta de medição foram identificadas as perguntas, com o objetivo de traduzir as metas em aspectos quantitativos que pudessem ser alvos de medição. A partir das questões, derivaram-se então as métricas. $\mathrm{O}$ quadro 4 apresenta o conjunto de métricas para a avaliação da qualidade da tradução de requisitos para casos de uso (U.C.).

\section{Quadro 4 Modelo GQM proposto}

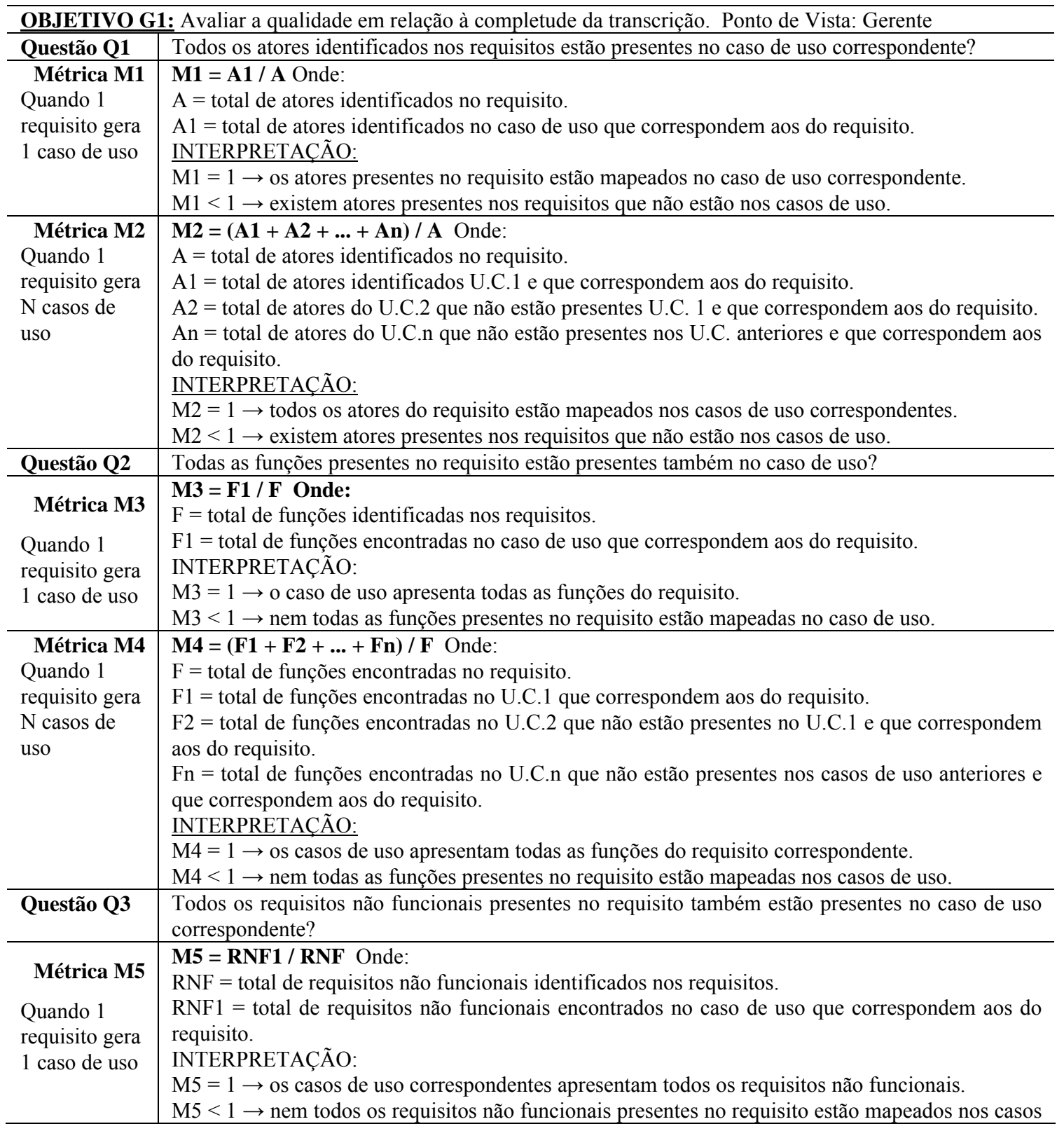

Anais do III Simpósio Brasileiro de Sistemas de Informação. Curitiba, PR, novembro de 2006. 


\begin{tabular}{|c|c|}
\hline & de uso. \\
\hline $\begin{array}{l}\text { Métrica M6 } \\
\text { Quando 1 } \\
\text { requisito gera } \\
\text { N casos de } \\
\text { uso }\end{array}$ & $\begin{array}{l}\text { M6 = (RNF1 + RNF2 + ... + RNFn) / RNF Onde: } \\
\text { RNF = total requisitos não funcionais (RNF) encontradas no requisito. } \\
\text { RNF1 = total de RNF encontrados no U.C.1 que correspondem aos do requisito. } \\
\text { RNF2 = total de RNF encontrados no U.C. } 2 \text { que não estão presentes nos U.C.1 e que } \\
\text { correspondem aos do requisito. } \\
\text { RNFn = total de RNF encontrados no U.C.n que não estão presentes nos casos de uso anteriores e } \\
\text { que correspondem aos do requisito. } \\
\text { INTERPRETAÇÃO: } \\
\text { M6 }=1 \rightarrow \text { os casos de uso apresentam todos os RNFs do requisito correspondente. } \\
\text { M6 }<1 \rightarrow \text { nem todos RNFs presentes no requisito estão mapeadas nos casos de uso. }\end{array}$ \\
\hline Questão Q4 & A transcrição de requisitos para caso de uso está completa? \\
\hline Métrica M7 & 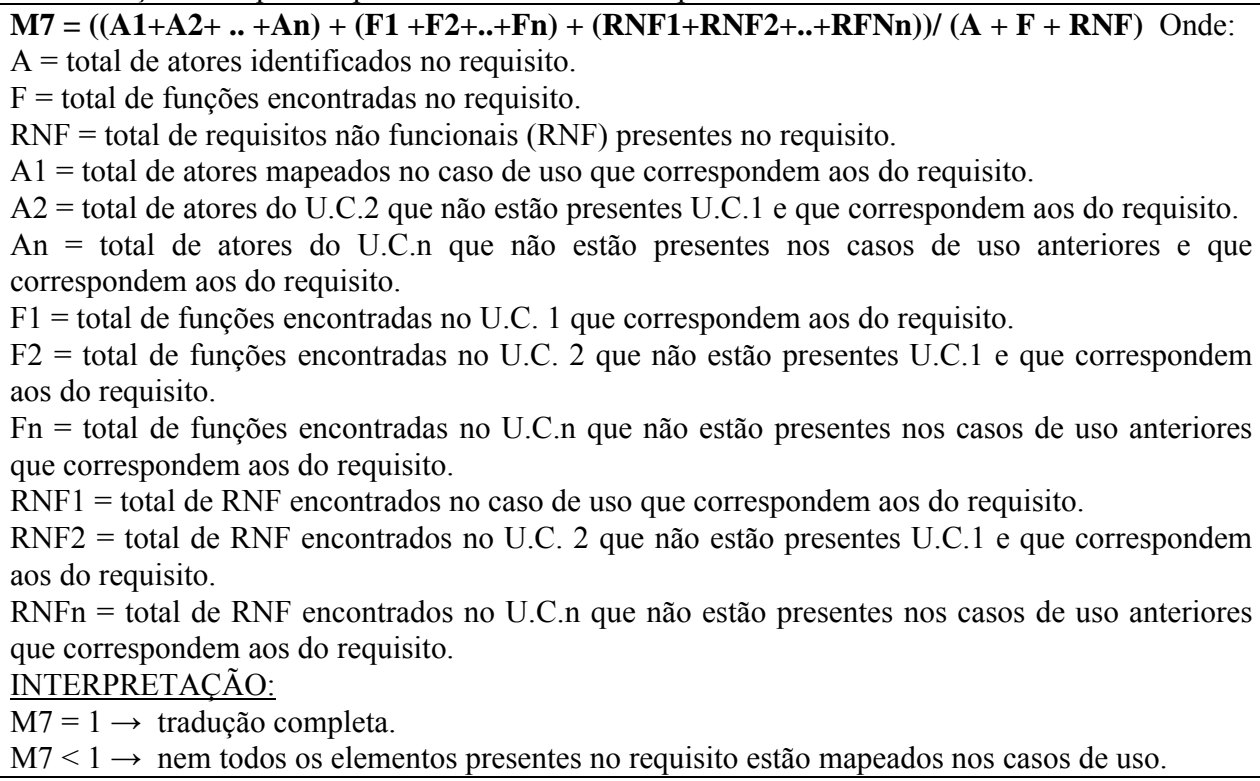 \\
\hline \multicolumn{2}{|c|}{ OBJETIVO G2: Avaliar a qualidade em relação à não ambigüidade da tradução. Ponto de Vista: Gerente } \\
\hline Questão Q5 & Os requisitos e casos de uso correspondentes possuem a mesma interpretação? \\
\hline $\begin{array}{l}\text { Métrica M8 } \\
\text { Quando } 1 \\
\text { requisito gera } \\
1 \text { caso de uso }\end{array}$ & $\begin{array}{l}\text { M8 = X } \\
\text { Onde } \mathrm{X}=0 \begin{array}{l}\text { os requisitos e os casos de uso correspondentes são ambíguos. } \\
1\end{array} \quad \text { os requisitos e os casos de uso correspondentes não são ambíguos. }\end{array}$ \\
\hline $\begin{array}{l}\text { Métrica M9 } \\
\text { Quando 1 } \\
\text { requisito gera } \\
\mathrm{N} \text { casos de } \\
\text { usos }\end{array}$ & $\begin{array}{l}\text { M9 }=\text { UCun / UC Onde: } \\
\text { UCun = número de casos de uso que tem a mesma interpretação do requisito. } \\
\text { UC = total de casos de uso gerados a partir do requisito. } \\
\text { INTERPRETAÇÃO: } \\
\text { M9 = } 1 \rightarrow \text { o requisito e o caso de uso correspondente têm a mesma interpretação. } \\
\text { M9 }<1 \rightarrow \text { o requisito e o caso de uso correspondente não possuem a mesma interpretação. }\end{array}$ \\
\hline \multicolumn{2}{|c|}{ OBJETIVO G3: Avaliar a qualidade em relação à consistência da tradução. Ponto de Vista: Gerente } \\
\hline$\overline{\text { Questão Q6 }}$ & Os requisitos e casos de uso correspondentes são consistentes entre si? \\
\hline $\begin{array}{l}\text { Métrica M10 } \\
\text { Quando } 1 \\
\text { requisito gera } \\
1 \text { caso de uso }\end{array}$ & $\begin{array}{l}\text { M10 = X } \\
\text { Onde X =0 } \\
1 \text { os requisitos e os casos de uso não são consistentes. } \\
1 \text { os requisitos e os casos de uso são consistentes. }\end{array}$ \\
\hline $\begin{array}{l}\text { Métrica M11 } \\
\text { Quando 1 } \\
\text { requisito gera } \\
\mathrm{N} \text { casos de } \\
\text { usos }\end{array}$ & $\begin{array}{l}\text { M11 }=\text { UCc } / \text { UC Onde: } \\
\text { UCc }=\text { número de casos de uso consistentes com o requisito. } \\
\text { UC }=\text { total de casos de uso gerados a partir do requisito. } \\
\text { INTERPRETAÇÃO: } \\
\text { M11 = } 1 \rightarrow \text { o requisito e o caso de uso correspondente estão consistentes. } \\
\text { M11 }<1 \rightarrow \text { o requisito e o caso de uso correspondente estão contraditórios. }\end{array}$ \\
\hline
\end{tabular}

A aplicação das métricas para avaliação da sua efetividade foi realizada através da análise de um SRS e seus respectivos casos de uso. Inicialmente, as métricas são aplicadas na tradução de maneira mais geral (métrica M7) e estas apontam falhas no mapeamento, porém não indicam quais os elementos estão com problemas na tradução. 
Posteriormente, aplicam-se as métricas para verificar a presença de cada elemento identificado, tais como: atores, funções e requisitos não funcionais (métricas M1, M2, M3, M4, M5, M6), com a finalidade de detectar qual elemento apresenta problemas na tradução, ou seja, qual elemento presente no requisito não é mapeado para o caso de uso. A seguir, as métricas que avaliam a consistência e ambigüidade (métricas M8, M9, M10 e M11) são aplicadas.

Os resultados obtidos com os estudos se mostraram satisfatórios, já que através das métricas foi possível detectar o foco do problema da tradução dos requisitos para casos de uso, permitindo a correção dos mesmos sem que fosse necessário analisar todos os documentos e todas as partes da tradução. Através desta aplicação constatamos também a facilidade do uso das métricas, bem como a facilidade de interpretá-las.

\section{Trabalhos relacionados}

O trabalho desenvolvido por [BELGAMO 2005] apresenta uma técnica de leitura que apóia a construção de casos de uso e a análise de documentos de requisitos. Utiliza o padrão SRS proposto por [IEEE 1998] e o template proposto por [COCKBURN 2001], ambos utilizados neste trabalho. $\mathrm{O}$ autor descreve os primeiros passos para encontrar os atores e relacioná-los aos seus objetivos, porém não explica de maneira clara como é feita a transcrição da especificação dos requisitos para especificação de casos de uso. Embora, tal limitação tenha sido encontrada, o trabalho contribuiu para a pesquisa na definição de alguns campos importantes que devam estar presentes no SRS e também nas diretrizes utilizadas para extrair os atores e seus objetivos de um SRS.

As ferramentas QuARS [FABBRINI 2001] e ARM [WILSON 1997] foram criadas para detectar automaticamente a qualidade da especificação de requisitos de software. A análise é feita através de técnicas sintáticas ou léxicas nas sentenças do SRS. Ambas possuem um modelo de qualidade que é composto de um jogo de atributos de qualidade para que os requisitos expressos em linguagem natural sejam avaliados [FABBRINI 2001]. As ferramentas citadas avaliam ou a qualidade dos requisitos ou a dos casos de uso e não fazem nenhuma abordagem no que se refere à correlação que existe entre requisitos e casos de uso. Mesmo com a presença de tais limitações, os trabalhos contribuíram para o esclarecimento de como é feita a avaliação de qualidade nos requisitos e como as métricas são aplicadas.

\section{Conclusão}

Especificar requisitos de elevada qualidade não é tarefa trivial. Por isto a necessidade da análise com o objetivo de capturar falhas. As métricas podem ajudar a capturar os defeitos e erros de requisitos permitindo as mudanças necessárias. O que se encontra na literatura é a análise do SRS ou dos casos de uso em separado.

A proposta apresentada neste artigo é de um modelo que permita a avaliação da qualidade da tradução de requisitos para casos de uso, com o objetivo de gerar documentos na fase de especificação de um sistema (SRS e casos de uso) com qualidade e consistência, bem como identificar a correlação existente entre eles. Tal aspecto contribui para a qualidade do produto final do software evitando os custos decorrentes de um produto implantado a partir de falhas de qualidade nos seus estágios iniciais.

Atualmente, as métricas estão sendo aplicadas em SRS de sistemas de maior complexidade para avaliar o modelo e as métricas, refinando a proposta. Os SRS 
utilizados são documentos de projetos desenvolvidos em uma grande empresa de desenvolvimento de software com atuação internacional.

\section{Referências}

BELGAMO, A.; FABBRI, S. (2005) "TUCCA: Técnica de Leitura para apoiar a Construção de Modelos de Casos de uso e a Análise de Documentos de Requisitos". In: XIX Simpósio Brasileiro de Engenharia de Software. Brasil.

COCKBURN, A. (2001) "Escrevendo Casos de Uso Eficazes". Addison-Wesley, 246p.

COSTELLO, R. and LIU, D. (1995) "Metrics for Requirements Engineering". Journal of Systems Software, pp. 39-63.

DAVIS, A., et al. (1993) "Identifying and Measuring Quality in a Software Requirements Specification." In: Proceedings of the 1st International Software Metrics Symposium, pages. 141-152, Baltimore.

FABBRINI, F., et al. (2001) "An automatic quality evaluation for natural language requirements". In: Proceedings of the 7th International Workshop on Requirements Engineering: Foundation for Software Quality REFSQ'01.

IEEE STD 830-1998. (1998) "IEEE Recommended Practice for Software Requirements Specifications". New York, USA: The Institute of Electrical and Electronics Engineers.

JACOBSON, I.; BOOCH, G. and RUMBAUGH, J. (1999) "The unified software development process", Addison-Wesley, $463 \mathrm{p}$.

MORA, M.and DENGER, C. (2003) "Requirements Metrics - An initial literature survey on measurement approaches for requirement specifications". Fraunhofer Institut Experimentelles Software Engineering, IESE-Report No. 096.03/E.

PRESSMAN, R. (2001) "Engenharia de Software", Mc Graw Hill, 888 p.

SOLINGEN, R. and BERGHOUT, E. (1999) "The Goal/Question/Metric Method: A Practical Guide for Quality Improvement of Software Development”. McGrawHill.

SOMMERVILLE, I. (2005) “Integrated Requirements Engineering: A Tutorial”. IEEE Software, pp. 16-23.

WILSON, W.; ROSENBERG, L. and HYATT, L. (1997) "Automated Analysis of Requirement Specifications". In: Proceedings of the Nineteenth International Conference on Software Engineering. Boston.

\footnotetext{
${ }^{i}$ Estudo realizado pelo Grupo de Pesquisa em Engenharia de Requisitos do PDTI, financiado pela Dell Computadores do Brasil Ltda. com recursos da Lei 8.248/91.
} 